\title{
Radiologic-Pathologic Correlation of Liver Tumors
}

\author{
Eric Lachance ${ }^{1} \bullet$ Jake Mandziuk $^{2} \bullet$ Consolato M. Sergi ${ }^{3} \bullet$ Justin Bateman $^{2} \bullet$ \\ Gavin Low ${ }^{1}$ \\ ${ }^{1}$ Department of Radiology and Diagnostic Imaging, University of Alberta, Edmonton, \\ Alberta, Canada; ${ }^{2}$ Department of Laboratory Medicine and Pathology, University of Alberta, \\ Edmonton, Alberta, Canada; ${ }^{3}$ Departments of Pediatrics, Laboratory Medicine and \\ Pathology, Stollery Children's Hospital, University of Alberta, Edmonton, AB, Canada.
}

Author for correspondence: Jake Mandziuk, Department of Laboratory Medicine and Pathology, University of Alberta, Edmonton, AB, Canada. Email: jmandziu@ualberta.ca Doi: https://doi.org/10.36255/exonpublications.livercancer.2021.ch5

\begin{abstract}
Radiologic and pathologic features of common and/or critical tumor or tumor-like diagnoses (lesions) of the liver are discussed within. Hepatocellular lesions (focal nodular hyperplasia, hepatocellular adenoma, hepatocellular carcinoma, and hepatoblastoma), biliary lesions (mucinous cystic neoplasm and intrahepatic cholangiocarcinoma), vascular mesenchymal lesions (cavernous hemangioma, epithelioid hemangioendothelioma, and hepatic angiosarcoma), and metastatic malignancies are the primary focus, although a more comprehensive list of lesions is also provided. Definitions, distributions, gross appearances and microscopic pathological features are introduced first, followed by radiologic correlation. Multiple imaging modalities are explored with an emphasis on those that provide the greatest value for the lesion under evaluation. A common understanding of the features of both diagnostic specialties will allow for high-quality correlation and subsequent high-quality patient care. Representative images highlighting important features are also presented.
\end{abstract}

Keywords: imaging; liver; pathology; radiology; tumors

In: Liver Cancer. Sergi CM. (Editor). Exon Publications, Brisbane, Australia. ISBN: 978-0-6450017-2-3; Doi: https://doi.org/10.36255/exonpublications.livercancer.2021

Copyright: The Authors.

License: This open access article is licenced under Creative Commons Attribution-NonCommercial 4.0 International (CC BY-NC 4.0) https://creativecommons.org/licenses/by-nc/4.0/ 


\section{INTRODUCTION}

This chapter is designed to introduce the reader to the radiologic and pathologic features of common and/or critical tumor or tumor-like diagnoses (lesions) within the liver. It is divided into hepatocellular lesions, biliary lesions, vascular mesenchymal lesions, and metastatic malignancies. However, a broad differential is important when approaching liver lesions, and a more comprehensive list of lesions is provided in Table 1 .

\section{TABLE 1 Categorization of Liver Lesions}

\section{Hepatocellular}

\begin{tabular}{|c|c|c|}
\hline Benign & Precursors & Malignant \\
\hline Focal Nodular Hyperplasia & Low-Grade Dysplastic Nodule & Hepatocellular Carcinoma \\
\hline Macroregenerative Nodule & High-Grade Dysplastic Nodule & Hepatoblastoma \\
\hline \multicolumn{3}{|l|}{ Hepatocellular Adenoma } \\
\hline \multicolumn{3}{|l|}{ Biliary } \\
\hline Benign & Precursors & Malignant \\
\hline Bile Duct Hamartoma & $\begin{array}{l}\text { Intraductal Papillary } \\
\text { Neoplasm }^{\mathrm{a}}\end{array}$ & $\begin{array}{c}\text { Intraductal Papillary Neoplasm with } \\
\text { Associated Invasive Carcinoma }{ }^{a}\end{array}$ \\
\hline Bile Duct Adenoma & Mucinous Cystic Neoplasm ${ }^{\mathrm{a}}$ & $\begin{array}{l}\text { Mucinous Cystic Neoplasm with } \\
\text { Associated Invasive Carcinoma }^{\text {a }}\end{array}$ \\
\hline Biliary Adenofibroma & & Cholangiocarcinoma \\
\hline \multicolumn{3}{|l|}{ Solitary Biliary Cyst } \\
\hline \multicolumn{3}{|l|}{ Mesenchymal } \\
\hline Benign & Intermediate & Malignant \\
\hline Cavernous Hemangioma & $\begin{array}{l}\text { Inflammatory Myofibroblastic } \\
\text { Tumor }\end{array}$ & Epithelioid Hemangioendothelioma \\
\hline Infantile Hemangioma & Solitary Fibrous Tumor & Angiosarcoma \\
\hline Angiomyolipoma & Kaposi Sarcoma & Embryonal Rhabdomyosarcoma \\
\hline Mesenchymal Hamartoma & & Undifferentiated Embryonal Sarcoma \\
\hline Other & & \\
\hline
\end{tabular}

Metastatic Malignancy

Infection/Abscess

Confluent Hepatic Fibrosis

Hydatid Cyst

Neuroendocrine Neoplasm

Lymphoma

a The $4^{\text {th }}$ edition of the WHO Classification of Tumors of the Digestive System in 2010 removed the terms biliary cystadenoma and biliary cystadenocarcinoma, refining into mucinous cystic neoplasms (MCN) and intraductal papillary neoplasm (IPN), "with associated invasive carcinoma" added, if present. Literature in the interval using the term biliary cystadenoma and/or cystadenocarcinoma may include MCN and/or IPN, with or without associated invasive carcinoma. 
Radiology plays an important and varied role, including screening, diagnosis, and treatment of liver lesions. Ultrasound plays an increasingly important role in the diagnosis of liver lesions, in part due to increased utilization of contrastenhanced ultrasound (CEUS), with clinical use in Europe, Asia, and Canada established and increasing use in the United States after FDA approval for abdominal use in 2016 (1). Magnetic resonance imaging (MRI) is also becoming increasingly important, with the ability to perform additional pulse sequence phases (for example, diffusion-weighted imaging [DWI]) and increased accuracy without increased exposure to ionizing radiation as in computed tomography (CT). Additionally, hepatobiliary gadolinium contrast agents such as gadoxetic acid (Primovist, Eovist) are increasingly used, adding accuracy in some instances compared to conventional extracellular gadolinium contrast agents. Nuclear medicine tests are utilized in select scenarios and not elaborated on in detail. Dual photon nuclear imaging tests, such as positron emission tomography with computed tomography (PET/CT), play an important role in the detection of extrahepatic (and to a lesser extent, intrahepatic) metastases, and traditional single-photon emission computed tomography (SPECT) nuclear imaging tests (for example, Tc-99m sulfur colloid) are largely of historical value. Interventional radiology is not elaborated on in this chapter but is invaluable in diagnosing liver lesions with image-guided biopsy and in treatment.

Pathology provides further diagnostic insight into material obtained through biopsy or surgical resection. Gross appearances guide sampling approaches, and microscopic examination can lead to a final diagnosis. In challenging cases or in metastatic disease, additional ancillary studies, including immunohistochemical (IHC) stains, help determine cell lineage and refine the diagnosis. If present, characteristic molecular alterations can define a diagnosis.

\section{HEPATOCELLULAR LESIONS}

Hepatocytes are the main functional epithelial cells of the liver and are arranged within hepatic plates supported by a fine reticulin fiber meshwork. The following sections discuss lesions involving hepatocytes and includes focal nodular hyperplasia, hepatocellular adenoma, hepatocellular carcinoma, and hepatoblastoma.

\section{Focal nodular hyperplasia}

Focal nodular hyperplasia (FNH) (Figure 1) is a non-neoplastic mass-forming lesion characterized by hyperplastic nodules, fibrous septae, and abnormal blood vessels within a region of altered hepatic blood flow (2-3). FNH is a solitary mass in three-quarters of cases with a right-lobe predilection; however, more than fifteen masses have been documented distributed throughout the liver (4). Grossly, FNH is a well-circumscribed and non-encapsulated bulging mass with a central stellate scar and radiating fibrous septae that create a multinodular appearance. Diameters are usually less than $5 \mathrm{~cm}$ but can exceed $10 \mathrm{~cm}$, and central scars are present approximately half of the time (5). Microscopically, nodules show cytologically benign hepatocytes arranged in hepatic plates up to two cells thick with preservation of the reticulin network. Dystrophic vessels are present in the central 

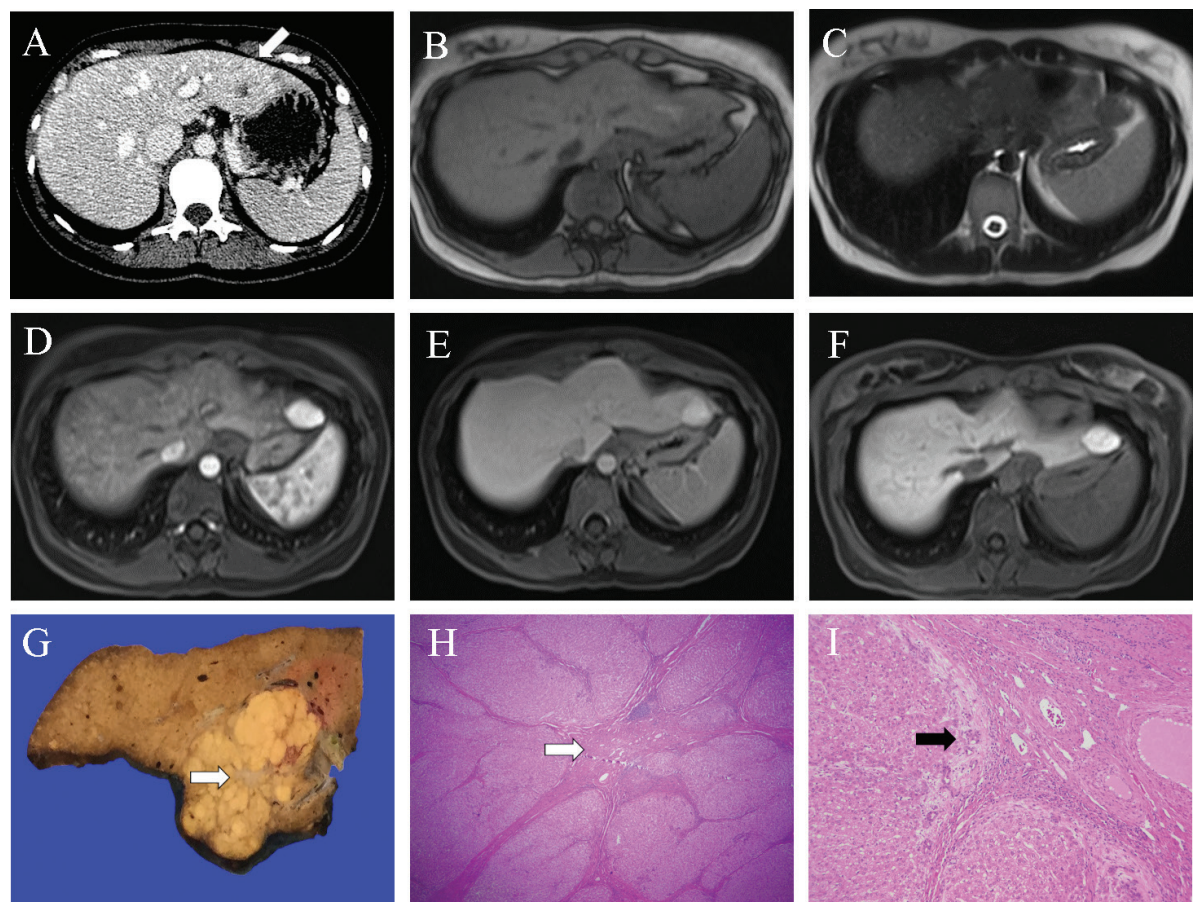

Figure 1. Representative images of focal nodular hyperplasia. A. On portal phase CT in liver windows the isoenhancing lesion with hypoenhancing central scar in segment $2 / 3$ is subtly appreciated (white arrow to the edge of lesion), subtly bulging the inner contour of the left lobe, a so-called "stealth" lesion. B and C. On MRI in a different patient, a similarly located segment $2 / 3$ lesion is isointense with the background liver on the T1 weighted image (B) and T2 weighted image (C). D and E. Following intravenous gadoxetic acid administration, the lesion shows homogenous hyperenhancement during the arterial (D) and portal (E) phases. F. On the hepatobiliary phase at 20 minutes, the lesion shows uniformly higher signal than the background liver. $\mathbf{G}$ and $\mathbf{H}$. A partial hepatectomy from a separate patient shows a well-circumscribed lesion with a central stellate scar (white arrow) and radiating fibrous septae, seen grossly $(\mathrm{G})$ and microscopically $(\mathrm{H})$. I. Ductular reaction (black arrow) is present at the interface of fibrous regions with nodules. Slides are stained with Hematoxylin and Eosin. Total image magnification: H - 25X; I - 100X.

scar, and a ductular reaction is seen at the interface between the fibrous septae and hyperplastic nodules. Normal bile ducts and portal tracts are absent. Glutamine synthetase demonstrates a characteristic 'map-like' pattern of staining by immunohistochemistry (6-7).

FNH can be subtle on unenhanced imaging, due to the benign hyperplastic tissue blending in with adjacent liver parenchyma, classically described as a "stealth lesion" (8). On ultrasound, the central scar may be indistinctly visualized; otherwise, FNH echogenicity is varied and nonspecific. However, contrastenhanced ultrasound, CT, or MRI is usually diagnostic. The central feeding artery with centrifugal flow may be seen as the characteristic "spoke-wheel" pattern on color Doppler, CEUS, and sometimes CT arterial phase, most commonly in larger lesions (9). FNH is typically a subtle hypoattenuating or isoattenuating 
homogenous lesion on unenhanced CT. On multiphasic CT, the arterial phase is most diagnostic for $\mathrm{FNH}$, with homogenous enhancement and hypoattenuating central scar, if present. On the portal-venous phase, enhancement is subdued, appearing mildly hyperattenuating or isoattenuating to the liver with the scar remaining hypoattenuating. On delayed phase imaging, the scar is typically hyperenhancing, with the remainder of the lesion isoattenuating.

MRI provides the most diagnostic characterization of FNH (with a specificity of 98\%) (10). FNH is Tl-weighted imaging (TlWI) isointense or slightly hypointense, and T2-weighted imaging (T2WI) isointense or slightly hyperintense. If present, the central scar is T1WI hypointense and T2WI hyperintense. Similar to CEUS and CT, during the arterial phase, FNH demonstrates intense homogenous enhancement (sparing the central scar), is relatively isointense on portal-venous phase (hypointense scar), and isointense on delayed phase (hyperintense scar). Gadoxetic acid-enhanced MRI is sensitive for FNH, showing a high signal on the hepatobiliary phase at 20 minutes, which aids in differentiation from other arterial enhancing liver lesions, such as adenomas (11).

\section{Hepatocellular adenoma}

Hepatocellular adenoma (HCA) (Figure 2) is a benign hepatocellular neoplasm, which may be solitary or multiple (2). Liver adenomatosis is defined by 10 or more HCAs and is diagnosed most frequently in patients with nodules greater than $5 \mathrm{~cm}$ (12). Hemorrhage is the first presentation in 15\% of patients and is associated with lesions greater than $5 \mathrm{~cm}$ (13). Grossly, HCAs are non-encapsulated and soft tan or red-brown masses that occur within a non-cirrhotic liver. Microscopically, the tumor is composed of benign hepatocytes arranged in plates up to two cells thick with unpaired arteries and an absence of portal tracts. Currently, there are four recognized genotypic-phenotypic subtypes: (i) HNF1 $\alpha$ inactivated HCA with marked steatosis and loss of liver fatty acid-binding protein expression by IHC, (ii) inflammatory HCA (JAK/STAT3 pathway activation) with inflammation, sinusoidal dilatation, ductular reaction, and diffuse expression of C-reactive protein and serum amyloid A by IHC, (iii) beta-catenin activated inflammatory HCA, and (iv) beta-catenin activated HCA. Beta-catenin activated HCAs may show atypical cytological and/or architectural features, suggesting transformation to hepatocellular carcinoma (HCC) (14). Evaluation of IHC stains may show nuclear beta-catenin expression along with over-expression of glutamine synthetase within these beta-catenin activated HCAs. Careful evaluation of a reticulin stain is essential to help exclude malignant transformation.

Imaging features of HCA are often varied, with slightly different characteristics depending on the subtype. Hemorrhage with possible subsequent calcification, fat, and encapsulation may be seen. Of note, HCAs $5 \mathrm{~cm}$ or larger are at higher risk for both hemorrhage and malignant transformation into HCC, and require close imaging follow up. Males with HCAs are often treated by prophylactic surgical resection, particularly for solitary or large lesions, as a result of the increased incidence of the beta-catenin subtype.

Ultrasound is nonspecific, demonstrating a well-circumscribed lesion with variable echogenicity, more often hyperechoic. CEUS may increase specificity, particularly showing centripetal arterial flow, differentiating HCA from FNH, which typically illustrate a centrifugal arterial flow. Multiphasic contrast-enhanced 

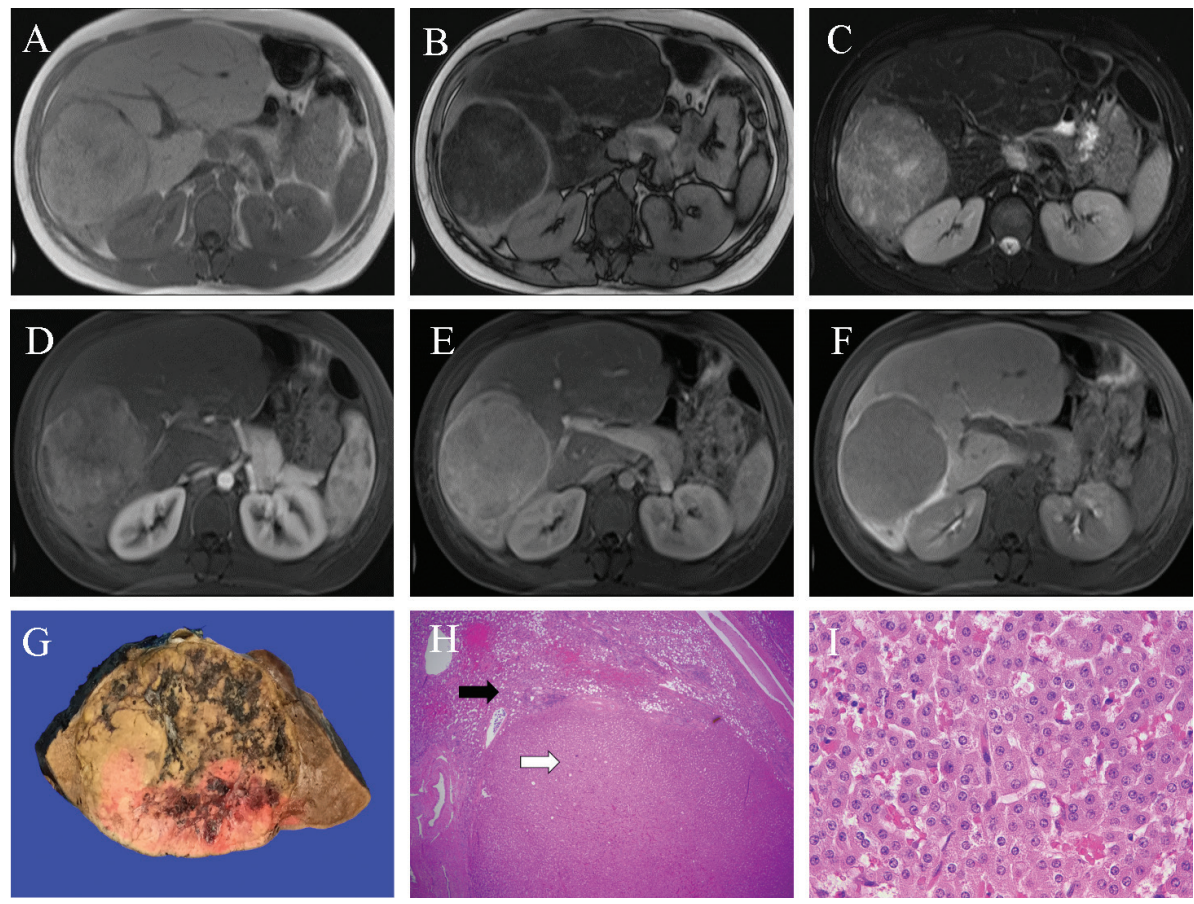

Figure 2. Representative images of hepatocellular adenoma. A and $\mathbf{B}$. The large lesion $(>5 \mathrm{~cm})$ in segments 5 and 6 shows signal loss on the opposed phase T1 weighted image (B) compared to the in-phase T1 weighted image (A) denoting microscopic fat content. C. The lesion shows heterogeneous high signal on the T2 weighted image. D, E, and F. Following gadolinium administration, the lesion shows heterogeneous arterial (D) and portal phase (E) hyperenhancement and delayed phase washout (F). An ancillary finding of an enhancing capsule is depicted in (E) and (F). G. A partial hepatectomy from a separate patient shows a tan and poorly defined lesion grossly, with areas of hemorrhage and necrosis. $\mathbf{H}$.

Microscopically the neoplastic lesion (white arrow) shows an absence of portal tracts and is notably different from the background parenchyma (black arrow). I. Benign hepatocytes are arranged in cell plates up to two cells thick. Slides are stained with Hematoxylin and Eosin. Total image magnification: $\mathrm{H}$ - 25X; I - 400X.

CT demonstrates a well-circumscribed mass, which is typically isoattenuating on the unenhanced phase, with heterogeneous arterial phase hyperenhancement, usually returning to near isoattenuation on portal-venous phase and delayed phase imaging. Following hyperenhancement, contrast washout (hypoenhancement on the portal-venous and delayed phases) may be seen in both CT and MRI, mimicking malignant lesions.

On MRI, T1WI and T2WI signal intensities are variable, depending on the presence of fat, hemorrhage, and calcification. A high signal on fat-saturated T1WI denotes the presence of intratumoral hemorrhage. Contrast-enhanced phases are similar to CT, with early arterial enhancement, and are typically isointense on portal-venous phase imaging. HCAs are predominantly hypointense on hepatobiliary phase gadoxetic acid imaging, an important distinction from FNH. Opposed phase T1WI and fat-saturated T1WI are helpful to demonstrate microscopic fat and macroscopic fat, respectively, a characteristic feature 
of HNF1 $\alpha$-inactivated HCAs. Conversely, inflammatory HCAs have a characteristic high signal peripheral rim on T2WI, attributed to sinusoidal dilatation, termed the "atoll sign" (15).

\section{Hepatocellular carcinoma}

Hepatocellular carcinoma (HCC) (Figure 3) is a malignant hepatocellular neoplasm, commonly occurring in the setting of underlying chronic liver disease (2). Traditionally, macroscopic patterns of HCC are described as single or multiple liver nodules, massive nodules that may involve multiple liver segments, and diffuse forms with multiple small nodules throughout the liver mimicking cirrhosis (16). Tumor nodules smaller than the main tumor and less than $2 \mathrm{~cm}$ away (separated by non-neoplastic parenchyma) are classified as satellite nodules and
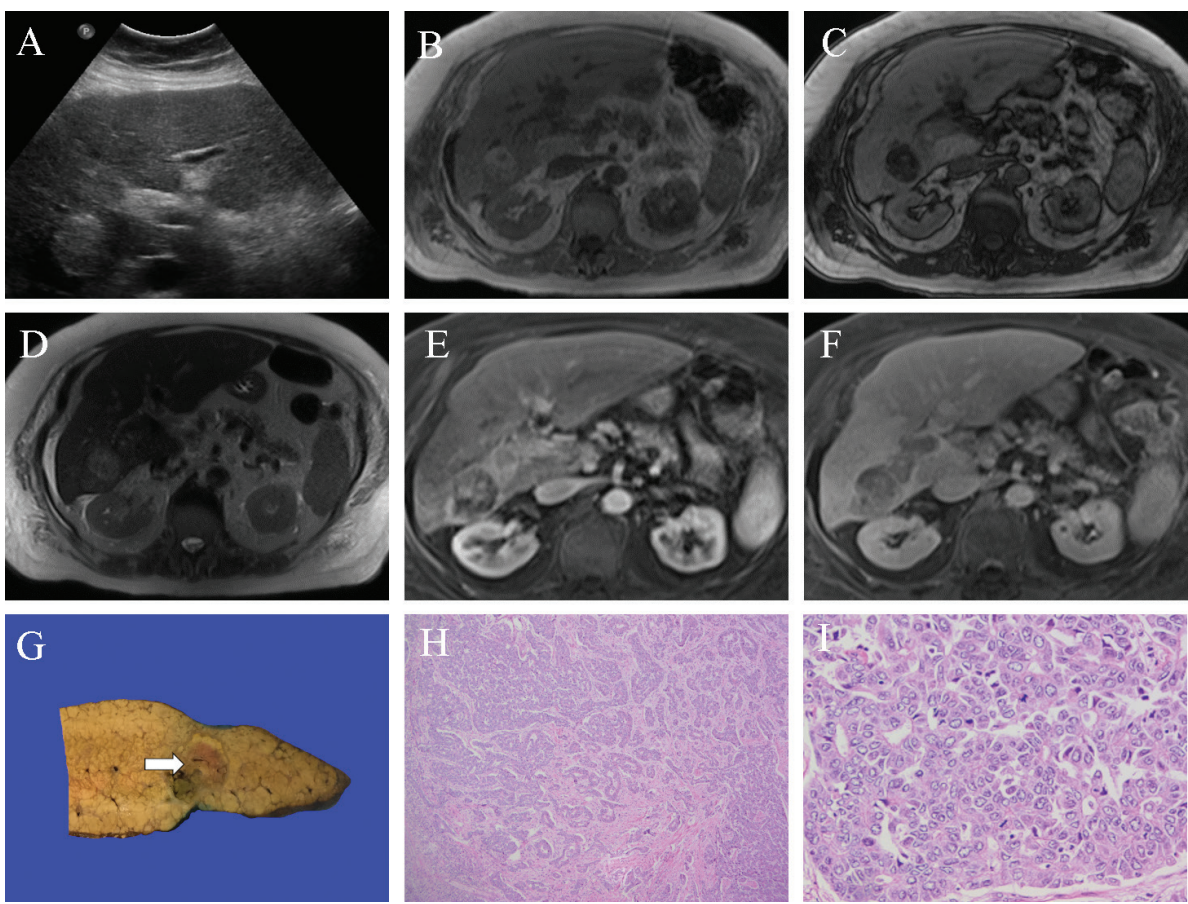

Figure 3. Representative images of hepatocellular carcinoma. A. Transverse ultrasound image of the liver demonstrates a new hyperechoic lesion in a cirrhotic liver, highly suspicious for HCC. B and C. The lesion in segment 6 shows signal loss on the opposed phase T1 weighted image (C) compared to the in-phase T1 weighted image (B) denoting microscopic fat content. D. The lesion shows heterogeneous high signal on the T2 weighted image. E and F. Following gadolinium administration, the lesion shows heterogeneous arterial enhancement (E) and portal phase wash-out (F). G. A representative slice of liver from an explant of a separate patient shows a soft variegated lesion grossly (white arrow), ranging from green to red-brown, embedded in a firm cirrhotic liver parenchyma. $\mathbf{H}$ and $\mathbf{I}$. There is a loss of normal hepatic architecture with trabecular and pseudoglandular growth patterns $(H)$ of malignant cells with enlarged vesicular nuclei and eosinophilic cytoplasm (I). Slides are stained with Hematoxylin and Eosin. Total image magnification: H - 25X; I - 400X. 
usually represent intrahepatic metastases. Nodules that are far from the primary tumor may represent either synchronous tumors or intrahepatic metastases (16). Involvement and spread through intrahepatic portal vein branches are common.

Grossly, HCC is soft with a variegated appearance ranging from green to light tan depending on bile and fat content respectively, and foci of hemorrhage or necrosis. Tumors may have a capsule of fibrotic and inflamed tissue and can be seen invading large vascular structures. Fibrolamellar and scirrhous subtypes may appear firmer and more fibrotic compared to conventional HCCs, and these features may mimic the central scar of FNH or even cholangiocarcinoma. Fibrolamellar carcinoma is a distinct lesion affecting younger adults without pre-existing cirrhosis or elevated serum AFP. It is typically a large mass with calcification, hemorrhage, or necrosis, can mimic FNH with a central scar, commonly metastasizes to lymph nodes and distant sites, and shows a DNAJB1-PRKACA fusion transcript (17-18).

Microscopically, HCC shows variable degrees of cytological and architectural atypia with thickened hepatic plates and disruption/loss of the reticulin framework. Hepatocellular differentiation is often apparent on hematoxylin and eosin (H\&E); however, IHC stains (Hep Par-1 and Arginase-1) are sometimes necessary for poorly differentiated tumors to establish hepatocellular origin. Furthermore, careful examination of reticulin stain and identification of abnormal expression of Glypican-3 IHC may be required in well-differentiated tumors to establish a diagnosis of carcinoma. Increased arterial blood flow leads to sinusoidal capillarization, which can be demonstrated by CD34 IHC. There are four predominant growth patterns including trabecular (70\%), solid (20\%), pseudoglandular (10\%), and macrotrabecular with cell plates greater than ten cells in thickness (1\%), although mixed patterns are common (19). Approximately 35\% of HCCs show distinct clinicopathologic subtypes, including steatohepatitic (most commonly in patients with underlying fatty liver disease), clear cell (cytoplasmic clearing secondary to glycogen accumulation), scirrhous (fibrosis in the majority of the tumor, separate small nests of tumor cells in thin trabeculae), and fibrolamellar (large polygonal hepatocytes with abundant cytoplasm and prominent nucleoli, pale bodies, thick collagenous bands in parallel orientations, and non-cirrhotic background liver) (20). Combined hepatocellular cholangiocarcinoma is a rare diagnosis with convincing HCC and cholangiocarcinoma morphologies present within the same tumor.

Precursor lesions of HCC are commonly identified in hepatectomy specimens and occasionally in biopsy specimens in patients with advanced chronic liver disease. These precursor lesions include dysplastic foci $(<1 \mathrm{~mm})$ and dysplastic nodules (approximately 5-15 mm) (21). Dysplastic foci are incidental findings and include small cell and large cell cytological change or small groups of hepatocytes with an otherwise clonal appearance (for example, iron free foci). Dysplastic nodules can be low- or high-grade, and the high-grade dysplastic nodules can mimic small $(<2 \mathrm{~cm}$ ) early HCCs. Features that favor the designation of HCC over a dysplastic nodule include a nodule-in-nodule growth pattern, stromal invasion, loss of reticulin, and expression of Glypican-3 by IHC. Low-grade dysplastic nodules require distinction from large regenerative nodules, although this distinction can be challenging and of limited significance in explanted livers (2).

Across imaging modalities, differentiation of small HCCs from regenerative or dysplastic nodules can be challenging in the setting of cirrhosis. Smaller lesions 
are typically homogenous, with larger lesions demonstrating variable heterogeneity secondary to the variable presence of fat, fibrosis, necrosis, hemorrhage, and calcification. The American College of Radiology (ACR) Liver Imaging and Reporting Data System (LI-RADS) version 2018 provides a validated set of standardized diagnostic criteria for HCC based on CT/MRI. It has become integrated into the American Association for the Study of Liver Diseases (AASLD) clinical practice guidelines (22-23). LI-RADS provides uniformity and improved accuracy in diagnostic reporting, including consistency in imaging work-up options and follow-up time intervals.

On ultrasound, small HCCs are usually hypoechoic, with larger lesions demonstrating variable echogenicity and heterogeneity. A thin hypoechoic pseudocapsule may be seen. Doppler may demonstrate vascularity and arteriovenous shunting. Increased arterialisation and progressive loss of the portal-venous vascular contribution results in a characteristic pattern on contrast-enhanced ultrasound, CT, and MRI of late arterial hyperenhancement with decreased enhancement relative to liver parenchyma (wash-out) on delayed phases, variably present on the portal-venous phase (24). Unenhanced CT has limited sensitivity for HCC, typically hypoattenuating if detected.

Contrast-enhanced MRI (extracellular or hepatobiliary gadolinium agents) shows significantly higher sensitivity for HCC than contrast-enhanced CT in the setting of cirrhosis ( $82 \%$ vs. $66 \%$ ) with similar specificities (91\% vs. 92\%) (25). Approximately 20\% of HCCs arise in non-cirrhotic livers, with contrast-enhanced MRI also demonstrating high sensitivity (91\%) and specificity (75-98\%) (26). HCC is usually TIWI isointense to hypointense, and T2WI heterogeneously intermediate to high signal. Late arterial hyperenhancement is typically homogenous for smaller lesions and more heterogeneous for larger lesions. Portal-venous phase wash-out is variable depending on size, with smaller lesions typically retaining a degree of perceptible enhancement and larger lesions demonstrating greater wash-out compared to the adjacent liver. With delayed phases, lesions typically demonstrate wash-out with residual enhancement in the pseudocapsule, if present. A recognized pitfall is that small $(<2 \mathrm{~cm})$ early HCCs may show a lack of delayed phase washout, and close imaging follow up of these lesions in the cirrhotic liver is required. DWI shows diffusion restriction in areas of high cellularity and cell membrane density (for example, malignancy) (27). DWI increases the sensitivity of conventional extracellular contrast-enhanced MRI, especially for small $(<2 \mathrm{~cm})$ HCCs (28). Sensitivity for HCC with gadoxetic acid contrastenhanced MRI is improved (95.2\%) when including wash-out criteria for the hepatobiliary phase (29).

\section{Hepatoblastoma}

Hepatoblastoma (HB) is a primary malignant liver neoplasm that recapitulates the developing fetal/embryonal liver with variable proportions of epithelial and mesenchymal elements and is the most common malignant liver tumor in children (30). HB is frequently a solitary mass; however, poor prognostic features include multifocality (20\%), the involvement of the vena cava or all three hepatic veins $(10 \%)$, the involvement of the portal bifurcation or both right and left portal veins (10\%), extrahepatic tumor extension (5\%), and tumor rupture (5\%) (31). Grossly, tumors are nodular or bosselated, and cut surfaces depend on tumor components, 
ranging from soft tan-brown in fetal patterns to gritty whitish speckles of osteoid in mesenchymal patterns. A variegated appearance can result from cystic degeneration, necrosis, or hemorrhage.

Microscopically, HBs are classified according to the International Pediatric Liver Tumors Consensus Classification of Liver Hepatoblastoma (32). Tumors can be either purely epithelial or contain a mixture of epithelial and mesenchymal components (spindle cells, osteoid, or cartilage) (32). Epithelial tumors may show a single or combination of histologic patterns including: fetal (characterized by trabecular growth of fetal-type hepatocytes, clear or finely granular cytoplasm depending on cytoplasmic glycogen content, variable degrees of mitotic activity, and nuclear pleomorphism), embryonal (primitive tubule formation, angulated nuclei, and high nuclear-to-cytoplasmic ratios), macrotrabecular (fetal or embryonal patterns in trabeculae greater than 5 cells thick), small cell undifferentiated (diffuse sheets or nests of cells with minimal cytoplasm, frequent apoptosis, mitotic activity, and necrosis), and cholangioblastic (formation of small ducts). The fetal pattern can be further subclassified into those with low-mitotic activity (well-differentiated), mitotically active (crowded fetal), and pleomorphic types. The small cell undifferentiated pattern can be further subdivided into SMARCB1 (INI1) positive and negative types with the help of IHC (2). Mixed epithelial and mesenchymal tumors are further subclassified into those with and without teratoid features. Given the wide variation in histological patterns, biopsies may not be representative of the tumor overall. Neoadjuvant chemotherapy can markedly alter the histologic appearance with changes including fibrosis, necrosis/hemorrhage, and the presence of osteoid-like tissue (30).

Ultrasound is commonly the first modality used when detecting HB, with further characterization by CT or MRI. MRI is advantageous in children, given the lack of radiation, and likely has increased sensitivity and specificity with gadoxetic acid MRI. Ultrasound appearances are dependent on the underlying histologic pattern. Generally, these lesions are lobular, well-circumscribed hypoechoic or heterogeneous lesions. Calcification, necrosis and vascular invasion may be seen. HB is typically heterogeneous and hypoattenuating to the liver parenchyma on CT. Speckled or amorphous calcification is seen in greater than $50 \%$ of lesions (33). Enhancement is typically less than that of the liver; however, peripheral arterial enhancement may be observed (33). On MRI, hepatoblastomas are typically heterogeneously T2WI hyperintense and T1WI hypointense. On contrast-enhanced MRI, HB is typically hypointense on the arterial, portal-venous, and delayed phases (34-35). The presence of a liver mass and elevated serum AFP in a child less than 4 years should alert the radiologist to the possibility of $\mathrm{HB}$.

\section{BILIARY LESIONS}

A network of bile canaliculi and a system of bile ducts located within portal tracts transports bile into larger intrahepatic ducts, exiting at the liver hilum. The following sections discuss lesions involving bile ducts and includes mucinous cystic neoplasm and intrahepatic cholangiocarcinoma. 


\section{Mucinous cystic neoplasm}

Mucinous cystic neoplasm (MCN) is a cyst-forming epithelial neoplasm of the liver that occurs almost exclusively in females. MCNs generally do not communicate with the biliary tree and show a characteristic subepithelial ovarian-type stroma, ranging from focal (8\%) to diffuse (36\%) (36). These two features can help differentiate it from other cystic neoplasms of the liver (for example, intraductal papillary neoplasm of the bile ducts). MCNs are uncommon hepatic cysts $(11 \%)$, frequently solitary, have an average size of $11 \mathrm{~cm}$ (range 5-23 cm), located predominantly in the left lobe (72\%), and rarely have associated carcinomas (6\%) (36). Grossly, MCNs are well-demarcated multiloculated cystic lesions with smooth inner surfaces containing mucinous, hemorrhagic, or serous fluid. Solid grey-white areas are concerning for invasive carcinoma. Microscopically, cyst walls are lined by either flattened epithelial cells or columnar/cuboidal cells with eosinophilic or mucinous cytoplasm and basally oriented nuclei and are delimited by a fibrous capsule. Low- and intermediate-grade dysplasia is common in MCNs, and cases must be thoroughly sampled and examined to exclude invasive adenocarcinoma, often with associated high-grade dysplasia (37). Estrogen and progesterone receptor IHC can be used to highlight the characteristic stroma in certain cases (2).

Radiology literature historically and still frequently describes MCNs of the liver and bile ducts as biliary cystadenomas and biliary cystadenocarcinomas. Across modalities (ultrasound, CT, MRI), MCNs are typically large, solitary, multilocular cystic lesions with smooth margins and internal septations (38). Varying mucin concentrations produce differing signal intensity within each locule of the multilocular cystic lesion; intermediate to high signal on T2WI, and low to high signal on T1WI. This may produce a "stained-glass" appearance, more commonly described in mucinous ovarian epithelial cystic neoplasms.

The presence of rapid growth, solid nodularity (particularly if enhancing), coarse calcification, irregular septations, and internal hemorrhage is suspicious for cystadenocarcinoma, although multiple studies have proven that imaging is not sufficiently diagnostic (39).

\section{Intrahepatic cholangiocarcinoma}

Intrahepatic cholangiocarcinoma (ICCA) (Figure 4) is a primary intrahepatic malignant neoplasm of epithelial cells with biliary differentiation. Two subtypes with unique clinicopathological features have been described: (i) large duct ICCA, arising from large perihilar intrahepatic bile ducts proximal to the right and left hepatic ducts, and (ii) small duct ICCA arising from small bile ducts or ductules in a peripheral location. Large duct and small duct subtypes account for $45 \%$ and $55 \%$ of all ICCAs, respectively (40), with $60 \%$ of all ICCAs being greater than $5 \mathrm{~cm}$ in size (41).

Grossly, large duct ICCAs often present as mass lesions around the larger caliber bile duct branches with invasion into the surrounding hepatic parenchyma. Some large duct ICCAs will not form a mass lesion but rather spread along bile ducts in a diffuse and longitudinal manner (referred to as a "periductal infiltrating" pattern of growth) (42). They may be found in association with macroscopically visible intraductal papillary neoplasms of the bile ducts, not discussed within 

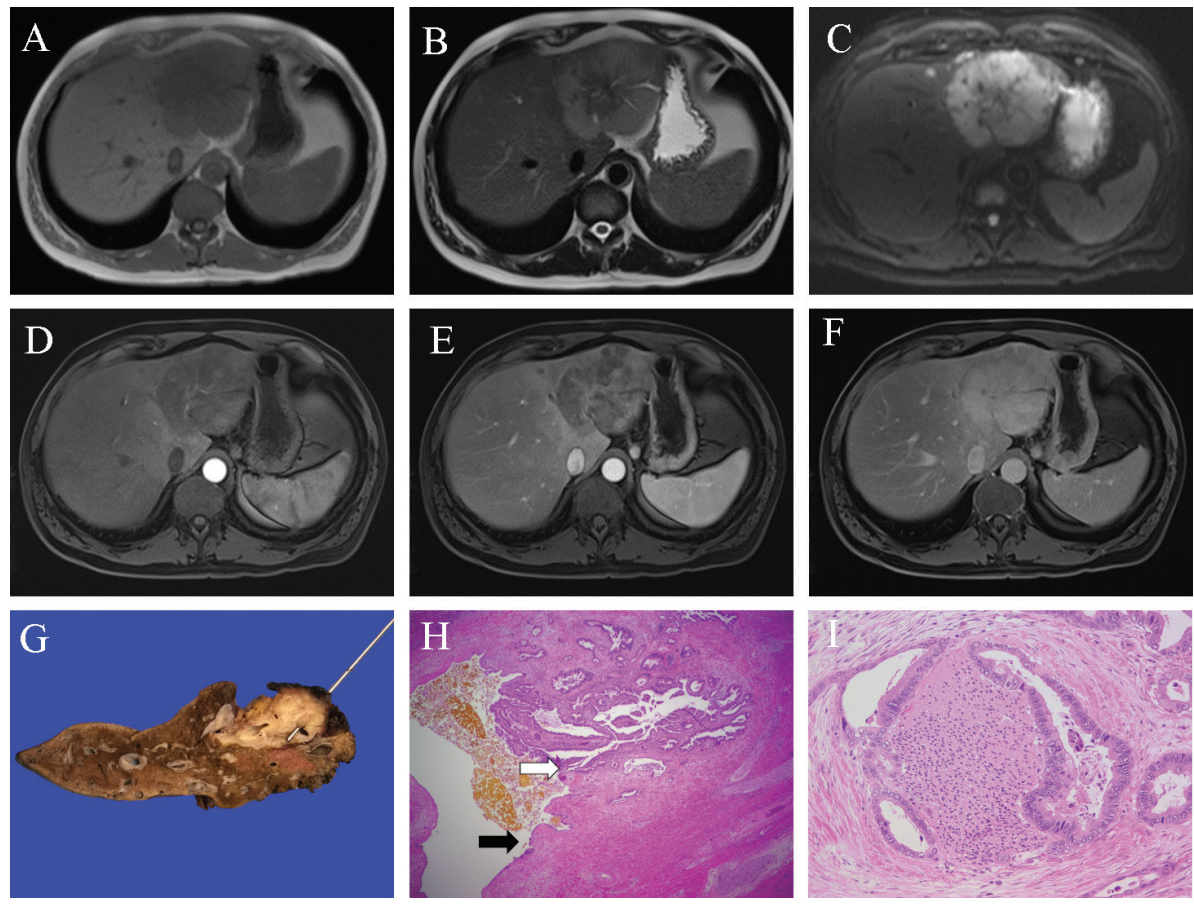

Figure 4. Representative images of intrahepatic cholangiocarcinoma. A and $\mathbf{B}$. The lesion shows low signal on the T1 weighted image (A) and intermediate to high signal on the T2 weighted image (B) with surrounding mild peripheral intrahepatic biliary dilatation. C. The lesion shows intense diffusion restriction on the $b=150 \mathrm{~s} / \mathrm{mm}^{2}$ image. D, E, and F. Following gadolinium administration, the lesion shows heterogeneous arterial (D) and portal phase (E) enhancement (predominantly in the periphery), with progressive centripetal filling-in, completely hyperenhancing on the delayed phase $(\mathrm{F})$ at 5 minutes. G. A partial hepatectomy from a separate patient shows a tan-white sclerosing lesion grossly, with extensive involvement of a probed large duct. H. Microscopically an invasive adenocarcinoma (white arrow) is seen arising from a large duct (black arrow). I. Well-formed glands are seen invading a nerve. Slides are stained with Hematoxylin and Eosin. Total image magnification: H - 25X; I - 200X.

this chapter. Small duct ICCAs typically present as a peripheral mass-forming lesion that is white-grey and nodular, often within a background of cirrhosis (62\% of cases) (40).

Microscopically, large duct ICCAs are invasive adenocarcinomas of tubular or solid growth patterns with extensive desmoplastic reaction, sclerosis of larger bile ducts, portal tract involvement, frequent perineural and lymphatic space invasion, and frequent lymph node metastasis. They may be found in association with microscopically visible biliary intraepithelial neoplasms, which are not discussed in this chapter. Small duct ICCAs show variable proportions of slit-like lumens in cord-like growth patterns, or distinct lumens in tubular growth patterns found replacing the hepatocytes in regenerative nodules. All ICCAs have small-tomedium-sized cuboidal or columnar cells, eosinophilic or vacuolated cytoplasm, small nuclei, and variably prominent nucleoli. The diagnosis of adenocarcinoma is typically readily made by morphology alone; however, poorly differentiated 
cases may lack apparent glandular differentiation and require IHC to exclude other primary neoplasms (for example, poorly differentiated HCC). Combined hepatocellular cholangiocarcinoma is a rare malignancy composed of both unequivocal HCC and ICCA components within the same tumor and may occasionally be encountered in biopsy specimens. ICCA does not show a specific morphology or IHC profile. Therefore, careful clinical and radiological correlation is essential to rule out a metastatic adenocarcinoma from another site.

On imaging, the mass-forming pattern of ICCA demonstrates a well-circumscribed, often large, lobulated mass frequently with satellite nodules. The periductal-infiltrating pattern demonstrates growth along bile ducts with an elongated, branching, spiculated appearance. Capsular retraction, more typical of the mass-forming pattern, is characteristic but not pathognomonic for ICCA. Upstream biliary ductal dilatation is typical but not specific.

Mass-forming ICCAs are typically hyperechoic on ultrasound if larger than $3 \mathrm{~cm}$ and isoechoic or hypoechoic if smaller (43). A peripheral hypoechoic rim is seen in approximately 35\% of cases (43). On unenhanced CT, they are typically hypoattenuating (43), and calcification may be present. They are typically heterogeneously T1WI hypointense and demonstrate a T2WI hyperintense peripheral rim with central hypodensity, which may be hyperintense in the setting of internal necrosis (43). The enhancement pattern on MRI and CT is typically arterial phase continuous rim-like enhancement (targetoid) with progressive, concentric centripetal fill-in on the portal-venous phase and persistent enhancement on delayed imaging (43).

Periductal-infiltrating ICCAs are typically enhancing on CT and MRI (43). Bile duct imaging, including endoscopic retrograde cholangiopancreatography (ERCP) and magnetic resonance cholangiopancreatography (MRCP), are highly sensitive and specific for bile duct narrowing and stricturing, which may be otherwise subtle. MRCP is $97 \%$ sensitive and $98 \%$ specific for the presence of obstruction (44).

Differentiating HCC from ICCA is important as locoregional treatments for HCC (for example, ablative or transcatheter therapy) are not effective for ICCA, and liver transplantation is a relative contraindication for ICCA due to the high risk of recurrence (45). A helpful finding is the presence of tumor thrombus, more commonly associated with HCC, while ICCA more commonly causes venous occlusion from extrinsic compression.

\section{VASCULAR MESENCHYMAL LESIONS}

Fine vascular sinusoids transport blood between hepatocytes from terminal branches of both the hepatic artery and hepatic portal vein, components of the portal tracts, to hepatic venules that drain into the hepatic vein. The following sections discuss lesions involving liver vasculature and includes cavernous hemangioma, epithelioid hemangioendothelioma, and hepatic angiosarcoma.

\section{Cavernous hemangioma}

Cavernous hemangioma $(\mathrm{CH})$ of the liver is a benign venous malformation with no malignant potential. CHs are most often solitary and are categorized according 
to size: small (less than $5 \mathrm{~cm}$ ), large $(5-10 \mathrm{~cm})$, and giant $(>10 \mathrm{~cm})$. Frequencies are approximately 30\%, 35\%, and 35\%, respectively (46). Grossly, CHs are wellcircumscribed, soft, spongy purple-red lesions located subcapsular or deep in the liver parenchyma. Microscopically, they consist of variably sized large vascular spaces lined by a bland epithelium and are embedded in a fibrous stroma. Thrombi may be present, and when extensive, can result in significant sclerosis of the lesion. Although well-circumscribed grossly, the tumor interface microscopically is irregular, with separate hemangioma-like vessels located $0.1-2 \mathrm{~cm}$ away from the lesion in $80 \%$ of cases (47).

As the most common benign liver tumor, $\mathrm{CH}$ s are frequently detected incidentally on imaging and can show a wide variety of atypical appearances. CHs are typically well-circumscribed and hyperechoic on ultrasound, with a well marginated lobulated contour that is better appreciated in larger CHs. Heterogeneity is more common with giant $\mathrm{CHs}$, although the lesion periphery typically remains echogenic (48). A central scar may be seen in a minority of giant CHs, mimicking $\mathrm{FNH}$, and calcification is rare, sometimes seen in the central scar. No central flow is demonstrated using color Doppler; however, peripheral vascularity can be present. A "reverse target" morphology with hyperechoic rim may be seen in a minority, with metastasis or HCC as the diagnosis of exclusion on a background of normal or cirrhotic liver parenchyma, respectively (49). Where CEUS is available, enhancement patterns parallel CT and MRI.

On CT, attenuation and enhancement across phases mirror blood pool (for example, the aorta), due to the underlying disorganized venous histology, with more rapid ("flash") filling and delayed/centripetal filling observed in small and giant CHs, respectively. The peripheral nodular contour with discontinuous enhancement is more conspicuous with large and giant CHs. The central scar, if present, is characteristically non-enhancing. MRI has the greatest sensitivity and specificity for diagnosing CH. On MRI, CHs are T1WI isointense or hypointense, with hypointensity typically observed in giant CHs. If present, the central scar is markedly T1WI hypointense. CHs are typically T2WI hyperintense due to the long $\mathrm{T} 2$ relaxation time in these lesions. MRI contrast enhancement patterns are similar to those described for CT; however, some CHs show slow enhancement with fill-in on delayed phase images, and rarely a centrifugal (inside-out) enhancement pattern (50).

Typically reserved for diagnostic clarification in patients unable to undergo MRI or contrast-enhanced studies, Tc-99m labelled RBC scans demonstrate photopenia or decreased activity relative to liver parenchyma on early dynamic scans with persistent filling of large and giant $\mathrm{CHs}$ on delayed imaging. This differentiates CHs from vascular tumors such as HCC, HCA, and FNH. When combined with multi-headed SPECT the positive predictive value approaches $100 \%$, with sensitivity markedly improving in larger lesions (20\% in lesions $0.5-0.9$ cm; $100 \%$ in lesions $>1.4 \mathrm{~cm})(51)$.

\section{Epithelioid hemangioendothelioma}

Epithelioid hemangioendothelioma (EHE) (Figure 5) of the liver is a malignant endothelial neoplasm. EHE is frequently multifocal (87\%), bilobar (81\%), and arises in the absence of chronic liver disease (52). Grossly, there is nodular or 

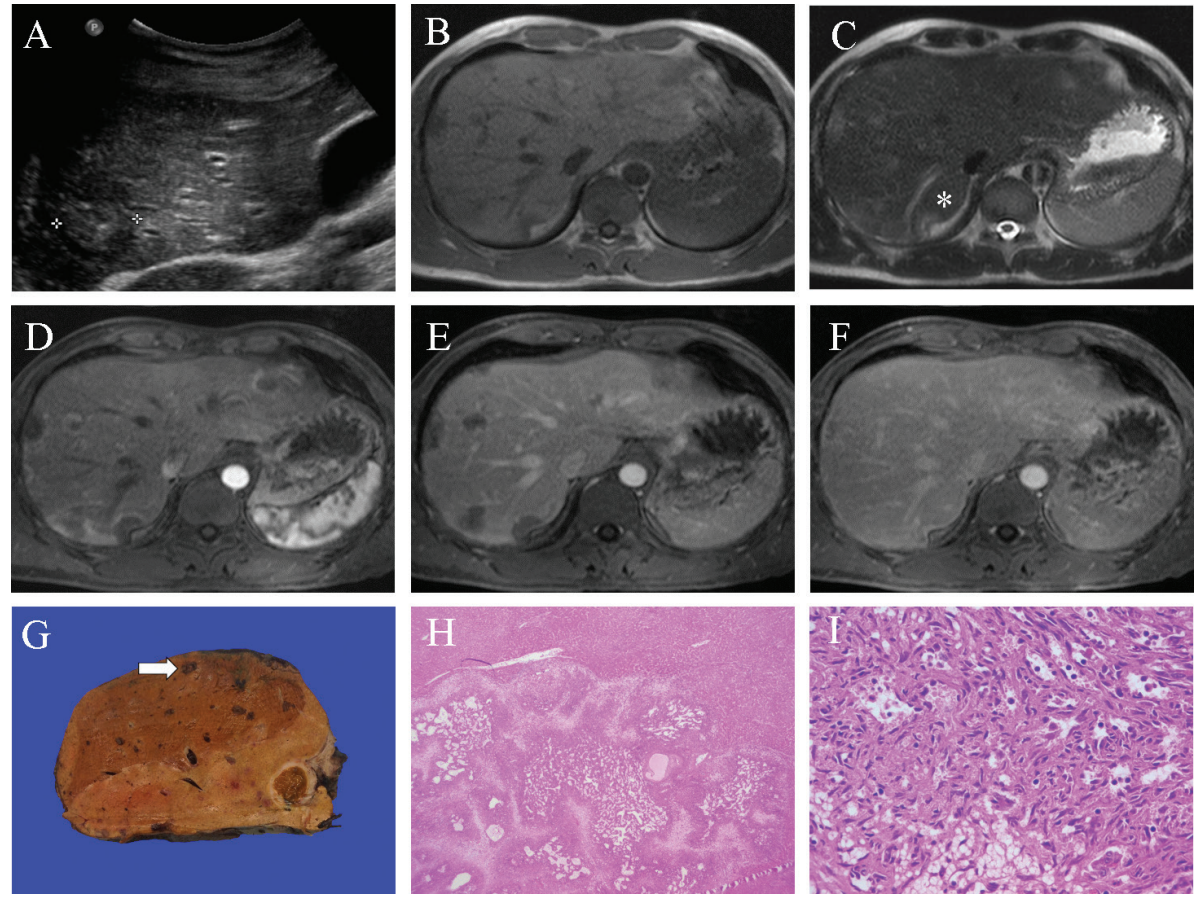

Figure 5. Representative images of hepatic epithelioid hemangioendothelioma. A. Transverse ultrasound image of the liver demonstrates a centrally hyperechoic lesion with a thin hypoechoic halo ("targetoid" appearance) in the right lobe of the liver with multifocal peripheral targetoid, heterogeneous and hypoechoic lesions involving both lobes (not shown). B and C. These lesions show low signal on the T1 weighted image (B) and intermediate to high signal on the T2 weighted image (C). One lesion in segment 7 at the subcapsular border is associated with focal capsular retraction $\left({ }^{*}\right)$. D, E, and F. Following gadolinium administration, the lesions show targetoid peripheral enhancement during the arterial (D) and portal (E) phases, and centripetal fill-in on the delayed phase (F). G. A representative slice of liver from a complete total hepatectomy of a separate patient shows multiple small red-brown lesions (white arrow) scattered throughout the parenchyma grossly. $\mathbf{H}$ and $\mathbf{I}$. Bland epithelioid and spindled cells (I) are embedded in a fibrous and myxohyaline background $(\mathrm{H})$. Slides are stained with Hematoxylin and Eosin. Total image magnification: $\mathrm{H}-25 \mathrm{X}$; I - 400X.

multinodular growth of firm, gray-white tumor. Microscopically, epithelioid or stellate spindled cells are arranged in cords, small nests, or single cells, and embedded in a fibrous or myxohyaline background. Nuclei show fine chromatin with small nucleoli, and the eosinophilic cytoplasm can show notable well-defined intracytoplasmic vacuoles/lumina of variable sizes. The mitotic rate is variable. WWRT1-CAMTA1 and YAP1-TFE3 translocations are present in approximately $80 \%$ and $5 \%$ of EHEs, respectively (53). A panel of IHC stains is typically required to confirm endothelial origin (for example, CD31 and ERG) and to exclude the possibility of carcinoma, although keratin stains may also be positive. CAMTA1 IHC stain is positive in a significant proportion of cases, given the associated gene rearrangement (2). 
EHE is most commonly identified as coalescent, peripheral, target-like hepatic nodules with capsular retraction on a normal liver parenchyma background. Calcification is uncommon. Ultrasound is nonspecific; nodule echogenicity is variable, often hypoechoic (54). Unenhanced CT is also nonspecific, demonstrating hypoattenuating nodules. A ring or target appearance is common on MRI and CT, with 2 or 3 rings of differing enhancement/intensity. A 3-ring lesion consists of a dominant central non-enhancing or delayed enhancing fibrous myxoid stroma, which may demonstrate necrosis, an enhancing inner peripheral ring of proliferating tumor cells, and an outer avascular non-enhancing rim of tumor infiltrating into sinusoids and portal branches (55-57). On T1WI, nodules are typically markedly hypointense centrally, with a less hypointense peripheral rim. On T2WI, small nodules are typically hyperintense, with larger lesions demonstrating a target pattern of central hyperintensity, a thin inner peripheral hypointense ring, and a hyperintense outer ring (56). Nodules may demonstrate a "Lollipop" sign; a well-defined, predominantly hypoenhancing nodule representing the spherical candy portion, and thrombosis within an associated vein, terminating smoothly at the edge or within the lesion rim, representing the stick (58).

\section{Hepatic angiosarcoma}

Hepatic angiosarcoma (HAS) is a primary malignant endothelial neoplasm. HAS is predominantly bilobar (80\%) and can show variable patterns of growth, including multinodular (40\%), solitary (20\%), dominant mass with satellite nodules (20\%), and diffusely infiltrating (20\%) (59). Grossly, tumors are poorly defined and heterogenous, with solid gray-white areas and hemorrhagic areas showing large blood-filled spaces. Microscopically, tumors also display heterogeneity. Wellformed anastomosing vascular channels alternate with solid sheets of tumor lacking vascular formation. Tumor cells can show high-grade features, including epithelioid or spindled cells with marked nuclear pleomorphism, hyperchromatic nuclei, and abundant mitoses. Infarct, necrosis, and fibrosis are common. At the periphery of the tumor, malignant cells characteristically grow along and replace endothelial cells present in pre-existing sinusoids. A high index of suspicion is required in some cases, along with a panel of IHC stains, to make a correct diagnosis. Epithelioid HAS in particular can mimic carcinomas, melanomas, and lymphomas by morphology alone, and therefore a panel of IHC stains is generally recommended, including markers of vascular differentiation such as CD31 and ERG (keratins and EMA may be positive) (2).

HAS demonstrates a variable appearance on radiologic imaging, reflecting the underlying pleomorphic histology. HAS is commonly heterogeneous and hypervascular, with multiplicity, multiorgan involvement, rapidly progressive growth, and a history of exposure to particular carcinogens (for example, vinyl chloride polymers) pointing toward the underlying diagnosis, which ultimately relies on histology. Hemorrhage, necrosis, and calcification may be seen. A background of liver cirrhosis or fibrosis is reported in approximately $40 \%$ of patients (60). On ultrasound, lesions are generally heterogeneous. On unenhanced CT, lesions are typically hypoattenuating, although heterogeneity may be seen, particularly in the setting of hemorrhage. On MRI, lesions are heterogeneous and may have an illdefined outline. They may be T1WI hypointense but can show high signal with 
intratumoral hemorrhage. On T2WI, HAS shows heterogeneous intermediate-tohigh signal intensity. On CT and MRI, HAS enhancement parallels blood pool, typically bizarre and disordered, with centrifugal and centripetal filling being the predominantly described progressive enhancement patterns (45, 60-61). Peripheral arterial enhancement is typically circumferential rather than nodular and discontinuous, as in CHs. Flash-filling of smaller lesions is also observed. In a multi-institutional review of 35 cases, arterial phase foci of hyperenhancement were seen in $89.7 \%$, with none demonstrating portal or hepatic vein invasion, which may help in distinguishing HAS from HCC (60). Rapid growth on serial imaging and ancillary findings such as lymph node or extrahepatic organ metastases are recognized features (45).

\section{METASTATIC MALIGNANCIES}

Metastatic malignancies are common in the liver (more common than primary malignancies) and frequently multifocal; however, solitary nodules and diffuse parenchymal involvement without discrete nodule formation is possible. Tumors are primarily carcinomas (92\%), of which adenocarcinomas account for the majority (75\%), but melanomas (2.4\%) and sarcomas (1\%) are also found (62). The most frequent primary sites of origin for adenocarcinomas are colorectal (46\%), pancreas (10\%), breast (8\%), and lung (4\%) (62). Gross and microscopic appearances differ based on the primary site of origin. Many cases of metastases involving the liver can be quickly sorted out based on a combination of tumor morphology/immunophenotype, clinical history, and radiology. Some cases, however, present a much greater challenge for pathologists, radiologists, and clinicians alike, and a multidisciplinary team-based approach can be tremendously helpful in determining the site of origin and/or appropriate management.

The radiologic appearance of metastatic malignancies also varies, with appearances mimicking those seen at the respective sites of primary malignancies. Common examples include hypovascular epithelial metastases (for example, colon, lung, and gastric), hypervascular metastases (for example, renal cell carcinoma, melanoma, thyroid, and neuroendocrine tumors), and cystic liver metastases (for example, mucinous or serous primary malignancies from the ovary, colon, and pancreas).

In comparison to the gold standard of resection or intraoperative ultrasound, conventional ultrasound has modest sensitivity for metastases (50-76\%), improving with contrast-enhancement (82-86\%) (63), showing similar sensitivities to contrast-enhanced CT and MRI (64-65). Ultrasound appearances are varied with the following typical appearances: hypoechoic in hypovascular metastases, centrally hyperechoic and peripherally hypoechoic (targetoid) in aggressive metastases, and hyperechoic in hypervascular metastases.

Unenhanced CT has poor sensitivity for most metastases, with high sensitivity for calcification, a nonspecific but common finding in mucinous lesions. Metastases are typically T1WI hypointense and T2WI hyperintense, heterogeneous in morphology, and may be associated with an ill-defined outline. Enhancement characteristics are reflective of the predominant hepatic arterial supply, appearing similar on CT and conventional extracellular 
contrast-enhanced MRI, with the latter demonstrating higher sensitivity and specificity. Arterial enhancement with wash-out on portal-venous and delayed phases is characteristic for malignant liver lesions (including metastases) with a sensitivity of $24.5 \%$ and specificity of $100 \%$ on MRI (66). Cystic metastases are typically non-enhancing.

On meta-analysis, gadoxetic acid-enhanced MRI was superior to contrastenhanced CT (sensitivity $86.9-100 \%$ vs. 51.8-84.6\%, specificity $80.2-98.0 \%$ vs. 77.2-98.0\%) (67). On gadoxetic acid-enhanced MRI, metastases are typically conspicuously hypointense on delayed phase imaging as a result of the lack of hepatocytes and biliary ducts. Additional sequences such as DWI may increase MRI sensitivity and specificity. 18F-fluorodeoxyglucose PET/CT (FDG PET/CT) is principally utilized for the detection of extrahepatic metastases, particularly in lymph nodes, with high background liver FDG uptake slightly limiting the detection of intrahepatic metastases, as seen when evaluating for colorectal cancer liver metastases, where MRI demonstrated superior sensitivity (84-86\%), when compared to FDG PET/CT (72\%) (68).

\section{CONCLUSION}

As research progresses, diagnostic criteria and diagnoses evolve. Novel techniques develop and characteristic molecular alterations are discovered. With an expansion of knowledge comes an increasing need for effective communication and feedback between diagnostic specialties. This chapter serves as a reference for diagnostic findings in common and/or critical liver lesions. Radiologic and pathologic correlation will continue to be necessary for providing patients, their families, and their clinical care teams accurate diagnoses to guide future management.

Conflict of interest: The authors declare no potential conflicts of interest with respect to research, authorship and/or publication of this article.

Copyright and permission statement: The authors confirm that the materials included in this chapter do not violate copyright laws. Where relevant, appropriate permissions have been obtained from the original copyright holder(s), and all original sources have been appropriately acknowledged or referenced.

\section{REFERENCES}

1. Barr RG, Wilson SR, Lyshchik A, McCarville B, Darge K, Grant E, et al. Contrast-enhanced UltrasoundState of the Art in North America: Society of Radiologists in Ultrasound White Paper. Ultrasound Q. 2020 Sep;36(4S):S1-S39. https://doi.org/10.1097/RUQ.0000000000000515

2. WHO Classification of Tumours Editorial Board. Digestive System Tumours, WHO Classification of Tumours. 5th ed. Lyon, France: International Agency for Research on Cancer; 2019. p. 221-244, 250-3, 466-7, 471-2.

3. Wanless IR, Mawdsley C, Adams R. On the pathogenesis of focal nodular hyperplasia of the liver. Hepatology. 1985;5(6):1194-200. https://doi.org/10.1002/hep.1840050622 
4. Nguyen BN, Fléjou JF, Terris B, Belghiti J, Degott C. Focal nodular hyperplasia of the liver: a comprehensive pathologic study of 305 lesions and recognition of new histologic forms. Am J Surg Pathol. 1999;23(12):1441-54. https://doi.org/10.1097/00000478-199912000-00001

5. Shen YH, Fan J, Wu ZQ, Ma ZC, Zhou XD, Zhou J, et al. Focal nodular hyperplasia of the liver in 86 patients. Hepatobiliary Pancreat Dis Int. 2007;6(1)52-7.

6. Bioulac-Sage P, Laumonier H, Rullier A, Cubel G, Laurent C, Zucman-Rossi J, et al. Over-expression of glutamine synthetase in focal nodular hyperplasia: a novel easy diagnostic tool in surgical pathology. Liver Int. 2009;29(3):459-65. https://doi.org/10.1111/j.1478-3231.2008.01849.x

7. Bioulac-Sage P, Cubel G, Taouji S, Scoazec JY, Leteurtre E, Paradis V, et al. Immunohistochemical markers on needle biopsies are helpful for the diagnosis of focal nodular hyperplasia and hepatocellular adenoma subtypes. Am J Surg Pathol. 2012;36(11):1691-9. https://doi.org/10.1097/ PAS.0b013e3182653ece

8. Buetow PC, Pantongrag-Brown L, Buck JL, Ros PR, Goodman ZD. Focal nodular hyperplasia of the liver: radiologic-pathologic correlation. Radiographics. 1996;16(2):369-88. https://doi.org/10.1148/ radiographics.16.2.8966294

9. Ungermann L, Eliás P, Zizka J, Ryska P, Klzo L. Focal nodular hyperplasia: Spoke-wheel arterial pattern and other signs on dynamic contrast-enhanced ultrasonography. Eur J Radiol. 2007;63(2): 290-4. https://doi.org/10.1016/j.ejrad.2007.01.026

10. Ronot M, Vilgrain V. Imaging of benign hepatocellular lesions: current concepts and recent updates. Clin Res Hepatol Gastroenterol. 2014;38(6):681-8. https://doi.org/10.1016/j.clinre.2014.01.014

11. Grazioli L, Bondioni MP, Haradome H, Motosugi U, Tinti R, Frittoli B, et al. Hepatocellular adenoma and focal nodular hyperplasia: value of gadoxetic acid-enhance MR imaging in differential diagnosis. Radiology. 2012;262(2):520-9. https://doi.org/10.1148/radiol.11101742

12. Barbier L, Nault JC, Dujardin F, Scotto B, Besson M, de Muret A, et al. Natural history of liver adenomatosis: A long-term observational study. J Hepatol. 2019;71(6):1184-92. https://doi.org/10.1016/j. jhep.2019.08.004

13. Laurent A, Dokmak S, Nault JC, Pruvot, FR, Fabre JM, Letoublon C, et al. European experience of 573 liver resections for hepatocellular adenoma: a cross-sectional study by the AFC-HCA-2013 study group. HPB (Oxford). 2016;18(9):748-55. https://doi.org/10.1016/j.hpb.2016.06.011

14. Gonzalez RS, Raza A, Propst R, Adeyi O, Bateman J, Sopha SC, et al. Recent Advances in Digestive Tract Tumors: Updates From the 5th Edition of the World Health Organization "Blue Book". Arch Pathol Lab Med. 2020. https://doi.org/10.5858/arpa.2020-0047-RA

15. Dharmana H, Saravana-Bawan S, Girgis S, Low G. Hepatocellular adenoma: imaging review of the various molecular subtypes. Clin Radiol. 2017;72(4):276-85. https://doi.org/10.1016/j.crad. 2016.12.020

16. Martins-Filho SN, Alves VAF. The strengths and weaknesses of gross and histopathological evaluation in hepatocellular carcinoma: a brief review. Surg Exp Pathol. 2019;2(1):23. https://doi.org/10.1186/ s42047-019-0047-6

17. Kim DH, Choi SH, Kim SY, Kim MJ, Lee SS, Byun JH. Gadoxetic Acid-enhanced MRI of Hepatocellular Carcinoma: Value of Washout in Transitional and Hepatobiliary Phases. Radiology. 2019;291(3):651-7. https://doi.org/10.1148/radiol.2019182587

18. Graham RP, Jin L, Knutson DL, Kloft-Nelson SM, Greipp PT, Waldburger N, et al. DNAJB1-PRKACA is specific for fibrolamellar carcinoma. Mod Pathol. 2015;28(6):822-9. https://doi.org/10.1038/ modpathol.2015.4

19. Rastogi A. Changing role of histopathology in the diagnosis and management of hepatocellular carcinoma. World J Gastroenterol. 2018;24(35):4000-13. https://doi.org/10.3748/wjg.v24.i35.4000

20. El Jabbour T, Lagana SM, Lee H. Update on hepatocellular carcinoma: Pathologists' review. World J Gastroenterol. 2019;25(14):1653-65. https://doi.org/10.3748/wjg.v25.i14.1653

21. International Working Party. Terminology of nodular hepatocellular lesions. Hepatology. 1995;22(3):983-93. https://doi.org/10.1016/0270-9139(95)90324-0

22. Elsayes KM, Kielar AZ, Elmohr MM, Chernyak V, Masch WR, Furlan A, et al. White paper of the Society of Abdominal Radiology hepatocellular carcinoma diagnosis disease-focused panel on 
LI-RADS v2018 for CT and MRI. Abdom Radiol. 2018;43(10):2625-42. https://doi.org/10.1007/ s00261-018-1744-4

23. Marrero JA, Kulik LM, Sirlin CB, Zhu AX, Finn RS, Abecassis MM, et al. Diagnosis, Staging, and Management of Hepatocellular Carcinoma: 2018 Practice Guidance by the American Association for the Study of Liver Diseases. Hepatology. 2018;68(2):723-50. https://doi.org/10.1002/hep.29913

24. Venkatesh SK, Chandan V, Roberts LR. Liver masses: a clinical, radiologic, and pathologic perspective. Clin Gastroenterol and Hepatol. 2014;12(9):1414-29. https://doi.org/10.1016/j.cgh.2013.09.017

25. Roberts LR, Sirlin CB, Zaiem F, Almasri J, Prokop LJ, Heimbach JK, et al. Imaging for the diagnosis of hepatocellular carcinoma: A systematic review and meta-analysis. Hepatology. 2018;67(1):401-21. https://doi.org/10.1002/hep.29487

26. Fischer MA, Raptis DA, Donati OF, Hunziker R, Schade E, Sotiropoulos GC, et al. MR imaging features for improved diagnosis of hepatocellular carcinoma in the non-cirrhotic liver: Multi-center evaluation. Eur J Radiol. 2015;84(10):1879-87. https://doi.org/10.1016/j.ejrad.2015.06.029

27. Taron J, Johannink J, Bitzer M, Nikolaou K, Notohamiprodjo M, Hoffmann R. Added value of diffusion-weighted imaging in hepatic tumors and its impact on patient management. Cancer Imaging. 2018;18(1):10. https://doi.org/10.1186/s40644-018-0140-1

28. Lan H, Lin G, Zhong W. A meta analysis of the added value of diffusion weighted imaging in combination with contrast enhanced magnetic resonance imaging for the diagnosis of small hepatocellular carcinoma lesser or equal to $2 \mathrm{~cm}$. Oncol Lett. 2020;20(3):2739-48. https://doi.org/10.3892/ ol.2020.11805

29. Kim DH, Choi SH, Kim SY, Kim MJ, Lee SS, Byun JH. Gadoxetic Acid-enhanced MRI of Hepatocellular Carcinoma: Value of Washout in Transitional and Hepatobiliary Phases. Radiology. 2019;291(3):651-57. https://doi.org/10.1148/radiol.2019182587

30. Ferrell LD, Kakar S, Terracciano LM, Wee A. Tumours and Tumour-like Lesions of the Liver. In: Burt AD, Ferrell LD, and Hübscher S, editors. Macsween's Pathology of the Liver. 7th edition. Philadelphia: Elsevier; 2018, p. 780-879. https://doi.org/10.1016/B978-0-7020-6697-9.00013-3

31. Meyers RL, Maibach R, Hiyama E, Häberle B, Krailo M, Rangaswami A, et al. Risk-stratified staging in pediatric hepatoblastoma: a unified analysis from the Children's Hepatic tumors International Collaboration. Lancet Oncol. 2017;18(1):122-31. https://doi.org/10.1016/S1470-2045(16)30598-8

32. López-Terrada D, Alaggio R, de Dávila MT, Czauderna P, Hiyama E, Katzenstein H, et al. Towards an international pediatric liver tumor consensus classification: proceedings of the Los Angeles COG liver tumors symposium. Mod Pathol. 2014;27(3):472-91. https://doi.org/10.1038/modpathol.2013.80

33. Chung EM, Lattin GE Jr, Cube R, Lewis RB, Marichal-Hernández C, Shawhan R, et al. From the archives of the AFIP: Pediatric liver masses: radiologic-pathologic correlation. Part 2. Malignant tumors. Radiographics. 2011;31(2):483-507. https://doi.org/10.1148/rg.312105201

34. Meyers AB, Towbin AJ, Geller JI, Podberesky DJ. Hepatoblastoma imaging with gadoxetate disodium-enhanced MRI-typical, atypical, pre- and post-treatment evaluation. Pediatr Radiol. 2012;42(7):859-66. https://doi.org/10.1007/s00247-012-2366-6

35. Baheti AD, Chapman T, Rudzinski E, Albert CM, Stanescu AL. Diagnosis, histopathologic correlation and management of hepatoblastoma: What the radiologist needs to know. Clin Imaging. 2018;52:273-9. https://doi.org/10.1016/j.clinimag.2018.08.009

36. Quigley B, Reid MD, Pehlivanoglu B, Squires MH 3rd, Maithel S, Xue Y, et al. Hepatobiliary Mucinous Cystic Neoplasms With Ovarian Type Stroma (So-Called "Hepatobiliary Cystadenoma/ Cystadenocarcinoma"): Clinicopathologic Analysis of 36 Cases Illustrates Rarity of Carcinomatous Change. Am J Surg Pathol. 2018;42(1):95-102. https://doi.org/10.1097/PAS.0000000000000963

37. Fujikura K, Akita M, Abe-Suzuki S, Itoh T, Zen Y. Mucinous cystic neoplasms of the liver and pancreas: relationship between KRAS driver mutations and disease progression. Histopathology. 2017;71(4):591-600. https://doi.org/10.1111/his.13271

38. Qian LJ, Zhu J, Zhuang ZG, Xia Q, Liu Q, Xu JR. Spectrum of multilocular cystic hepatic lesions: CT and MR imaging findings with pathologic correlation. Radiographics. 2013;33(5):1419-33. https:// doi.org/10.1148/rg.335125063 
39. Mavilia MG, Pakala T, Molina M, Wu GY. Differentiating Cystic Liver Lesions: A Review of Imaging Modalities, Diagnosis and Management. J Clin Transl Hepatol. 2018;6(2):208-16. https://doi. org/10.14218/JCTH.2017.00069

40. Akita M, Fujikura K, Ajiki T, Fukumoto T, Otani K, Azuma T, et al. Dichotomy in intrahepatic cholangiocarcinomas based on histologic similarities to hilar cholangiocarcinomas. Mod Pathol. 2017;30(7):986-97. https://doi.org/10.1038/modpathol.2017.22

41. Bagante F, Spolverato G, Merath K, Weiss M, Alexandrescu S, Marques HP, et al. Intrahepatic cholangiocarcinoma tumor burden: A classification and regression tree model to define prognostic groups after resection. Surgery. 2019;166(6):983-90. https://doi.org/10.1016/j.surg.2019.06.005

42. Uno M, Shimada K, Yamamoto Y, Nara S, Esaki M, Sakamoto Y, et al. Periductal infiltrating type of intrahepatic cholangiocarcinoma: a rare macroscopic type without any apparent mass. Surg Today. 2012;42(12):1189-94. https://doi.org/10.1007/s00595-012-0145-5

43. Chung YE, Kim MJ, Park YN, Choi JY, Pyo JY, Kim YC, et al. Varying appearances of cholangiocarcinoma: radiologic-pathologic correlation. Radiographics. 2009;29(3):683-700. https://doi.org/ $10.1148 / \mathrm{rg} .293085729$

44. Romagnuolo J, Bardou M, Rahme E, Joseph L, Reinhold C, Barkun AN. Magnetic resonance cholangiopancreatography: a meta-analysis of test performance in suspected biliary disease. Ann Intern Med. 2003;139(7):547-57. https://doi.org/10.7326/0003-4819-139-7-200310070-00006

45. Ho AK, Girgis S, Low G. Uncommon liver lesions with multimodality imaging and pathology correlation. Clin Radiol. 2018;73(2):191-204. https://doi.org/10.1016/j.crad.2017.07.016

46. Di Carlo I, Koshy R, Al Mudares S, Ardiri A, Bertino G, Toro A. Giant cavernous liver hemangiomas: is it the time to change the size categories? Hepatobiliary Pancreat Dis Int. 2016;15(1):21-9. https:// doi.org/10.1016/S1499-3872(15)60035-2

47. Kim GE, Thung SN, Tsui WM, Ferrell LD. Hepatic cavernous hemangioma: underrecognized associated histologic features. Liver Int. 2006;26(3):334-8. https://doi.org/10.1111/j.1478-3231.2005.01226.x

48. Moody AR, Wilson SR. Atypical hepatic hemangioma: a suggestive sonographic morphology. Radiology. 1993;188(2):413-7. https://doi.org/10.1148/radiology.188.2.8327687

49. Wu S, Zuo D, Hong Y. The Prevalence of Hyperechoic Rim in Solid Focal Liver Lesions and Its Implication. J Diagn Med Sonogr. 2018;35(1):3-8. https://doi.org/10.1177/8756479318798365

50. Mathew RP, Sam M, Raubenheimer M, Patel V, Low G. Hepatic hemangiomas: the various imaging avatars and its mimickers. Radiol Med. 2020;125(9):801-15. https://doi.org/10.1007/ s11547-020-01185-z

51. Ziessman HA, Silverman PM, Patterson J, Harkness B, Fahey FH, Zeman RK, et al. Improved detection of small cavernous hemangiomas of the liver with high-resolution three-headed SPECT. J Nucl Med. 1991;32(11):2086-91.

52. Mehrabi A, Kashfi A, Fonouni H, Schemmer P, Schmied BM, Hallscheidt P, et al. Primary malignant hepatic epithelioid hemangioendothelioma: a comprehensive review of the literature with emphasis on the surgical therapy. Cancer. 2006;107(9):2108-21. https://doi.org/10.1002/cncr.22225

53. Patel NR, Salim AA, Sayeed H, Sarabia SF, Hollingsworth F, Warren M, et al. Molecular characterization of epithelioid haemangioendotheliomas identifies novel WWTR1-CAMTAl fusion variants. Histopathology. 2015;67(5):699-708. https://doi.org/10.1111/his.12697

54. Mehrabi A, Kashfi A, Fonouni H, Schemmer P, Schmied BM, Hallscheidt P, et al. Primary malignant hepatic epithelioid hemangioendothelioma. Cancer. 2006;107(9):2108-21. https://doi.org/10.1002/ cncr. 22225

55. Mamone G, Miraglia R. The "Target sign" and the "Lollipop sign" in hepatic epithelioid hemangioendothelioma. Abdom Radiol (NY). 2019;44(4):1617-20. https://doi.org/10.1007/s00261-018-1820-9

56. Paolantonio P, Laghi A, Vanzulli A, Grazioli L, Morana G, Ragozzino A, et al. MRI of hepatic epithelioid hemangioendothelioma (HEH). J Magn Reson Imaging. 2014;40(3):552-8. https://doi.org/10.1002/ jmri.24391

57. Makhlouf HR, Ishak KG, Goodman ZD. Epithelioid hemangioendothelioma of the liver: a clincicopathologic study of 137 cases. Cancer. 1999;85(3):562-82. https://doi.org/10.1002/ (SICI)1097-0142(19990201)85:3<562::AID-CNCR7>3.0.CO;2-T 
58. Alomari AI. The lollipop sign: A new cross-sectional sign of hepatic epithelioid hemangioendothelioma. Eur J Radiol. 2006;59(3):460-4. https://doi.org/10.1016/j.ejrad.2006.03.022

59. Wilson GC, Lluis N, Nalesnik MA, Nassar A, Serrano T, Ramos E, et al. Hepatic Angiosarcoma: A Multi-institutional, International Experience with 44 Cases. Ann Surg Oncol. 2019;26(2):576-82. https://doi.org/10.1245/s10434-018-7062-9

60. Pickhardt PJ, Kitchin D, Lubner MG, Ganeshan DM, Bhalla S, Covey AM. Primary hepatic angiosarcoma: multi-institutional comprehensive cancer centre review of multiphasic CT and MR imaging in 35 patients. Eur Radiol. 2015;25(2):315-22. https://doi.org/10.1007/s00330-014-3442-0

61. Koyama T, Fletcher JG, Johnson CD, Kuo MS, Notohara K, Burgart LJ. Primary hepatic angiosarcoma: findings at CT and MR imaging. Radiology. 2002;222(3):667-73. https://doi.org/10.1148/ radiol.2223010877

62. de Ridder J, de Wilt JHW, Simmer F, Overbeek L, Lemmens V, Nagetegaal I. Incidence and origin of histologically confirmed liver metastases: an explorative case-study of 23,154 patients. Oncotarget. 2016;7(34):55368-76. https://doi.org/10.18632/oncotarget.10552

63. Albrecht T. Detection and Characterisation of Liver Metastases. In: Lencioni R, editor. Enhancing the Role of Ultrasound with Contrast Agents. Milano: Springer; 2006. p. 53-67. https://doi. org/10.1007/88-470-0476-4_4

64. Dietrich CF, Nolsøe CP, Barr RG, Berzigotti A, Burns PN, Cantisani V, et al. Guidelines and Good Clinical Practice Recommendations for Contrast-Enhanced Ultrasound (CEUS) in the Liver-Update 2020 WFUMB in Cooperation with EFSUMB, AFSUMB, AIUM, and FLAUS. Ultrasound Med Biol. 2020;46(10):2579-2604. https://doi.org/10.1016/j.ultrasmedbio.2020.08.015

65. Dietrich CF, Kratzer W, Strobe D, Danse E, Fessl R, Bunk A, et al. Assessment of metastatic liver disease in patients with primary extrahepatic tumors by contrast-enhanced sonography versus $\mathrm{CT}$ and MRI. World J Gastroenterol. 2006;12(11):1699-705. https://doi.org/10.3748/wjg.v12.i1 1.1699

66. Mahfouz AE, Hamm B, Wolf KJ. Peripheral washout: a sign of malignancy on dynamic gadoliniumenhanced MR images of focal liver lesions. Radiology. 1994;190(1):49-52. https://doi.org/10.1148/ radiology.190.1.8259426

67. Vreugdenburg TD, Ma N, Duncan JK, Riitano D, Cameron AL, Maddern GJ. Comparative diagnostic accuracy of hepatocyte-specific gadoxetic acid (Gd-EOB-DTPA) enhanced MR imaging and contrast enhanced CT for the detection of liver metastases: a systematic review and meta-analysis. Int J Colorectal Dis. 2016;31(11):1739-49. https://doi.org/10.1007/s00384-016-2664-9

68. Sivesgaard K, Larsen LP, Sørensen M, Kramer S, Schlander S, Amanavicius N, et al. Diagnostic accuracy of CE-CT, MRI and FDG PET/CT for detecting colorectal cancer liver metastases in patients considered eligible for hepatic resection and/or local ablation. Eur Radiol. 2018;28(11):4735-47. https:// doi.org/10.1007/s00330-018-5469-0 